\title{
A study on the fracture toughness of seawater-absorbed carbon nanotube/epoxy/basalt composites
}

\author{
Man Tae Kim ${ }^{1}$, Kyong Yop Rhee', , Hyun Ju Kim³ and Dong Ho Jung ${ }^{3}$ \\ ${ }^{1}$ Gumi Electronics \& Information Technology Research Institute, Gumi 730-853, Korea \\ ${ }^{2}$ School of Mechanical Engineering, Kyung Hee University, Yongin 446-701, Korea \\ ${ }^{3}$ Maritime and Ocean Engineering Research Institute, Korea Institute of Ocean Science and Technology, Daejeon 305-600, Korea
}

\section{Article Info \\ Received 31 May 2013 \\ Accepted 27 June 2013 \\ *Corresponding Author \\ E-mail: rheeky@khu.ac.kr}

\section{Open Access}

DOI: http://dx.doi.org/

10.5714/CL.2013.14.3.190

This is an Open Access article distributed under the terms of the Creative Commons Attribution Non-Commercial License (http://creativecommons.org/licenses/ by-nc/3.0/) which permits unrestricted non-commercial use, distribution, and reproduction in any medium, provided the original work is properly cited.

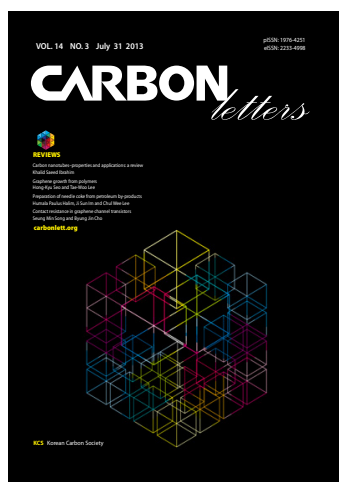

http://carbonlett.org

pISSN: 1976-4251

elSSN: 2233-4998

Copyright $\odot$ Korean Carbon Society

\begin{abstract}
It has been demonstrated in a previous study that carbon nanotube (CNT)/epoxy/basalt composites produce better flexural properties than epoxy/basalt composites. In this study, mode I fracture tests were conducted using CNT/epoxy/basalt composites with and without seawater absorption in order to investigate the effect of the seawater absorption on the mode I fracture toughness $\left(\mathrm{G}_{\mathrm{Ic}}\right)$ of the CNT/epoxy/basalt composites. The results demonstrated that the compliance of the seawater-absorbed specimen was larger than that of the dry specimen at the same crack length, while the opposite result was obtained for the fracture load. The $\mathrm{G}_{\text {Ic }}$ value of the seawater-absorbed CNT/epoxy/basalt composites was approximately $20 \%$ lower than that of the dry CNT/epoxy/basalt composites.
\end{abstract}

Key words: carbon nanotube/epoxy/basalt composites, seawater absorption, fracture toughness

\section{Introduction}

It is well-known that basalt fibers do not have toxic reactions with water and are noncombustible. When in contact with other chemicals, basalt fibers do not produce chemical reactions that may damage health or the environment. Furthermore, they have good mechanical and thermal properties and can have various applications as reinforcing materials in structural and constructional composite materials [1].

Accordingly, many studies have been conducted to investigate the mechanical properties of basalt fiber reinforced polymer matrix composites [2-5]. Recently, carbon nanotubes (CNTs) have been used as reinforcing materials in the polymer matrices because the addition of CNTs can improve the mechanical, electrical, and thermal properties of the conventional fiber-reinforced composites [6-8]. For example, Ashrafi et al. [9] investigated the effect of the addition of single-walled CNTs (SWCNTs) on the impact, compression, and fracture toughness of carbon/epoxy laminates. They reported that the addition of SWCNTs significantly improved the impact resistance and fracture toughness of the carbon/epoxy laminates. Furthermore, Kim et al. [10] investigated the effects of CNT modification on the fracture behavior of CNT/epoxy/basalt composites. Their results demonstrated that the fracture toughness of silane-treated CNT/epoxy/basalt composites was $40 \%$ greater than that of the acid-treated CNT/epoxy/basalt composites. However, an important issue for polymeric composites is that their mechanical properties degrade when exposed to seawater. To date, little research has been undertaken to investigate the fracture behavior of seawater-absorbed CNT/epoxy/basalt composites.

In the present study, the effects of seawater absorption on the fracture properties of CNT/ epoxy/basalt composites were investigated. Fracture tests were conducted using CNT/epoxy/basalt composites with and without seawater absorption. 


\section{Experimental}

The reinforcing materials that were used were woven-type basalt fibers (EcoB4-F260, Secotech, Korea) with an area density of $260 \mathrm{~g} / \mathrm{m}^{2}$ and multi-walled CNTs (MWCNTs; CM-95, Hanhwa Nanotech, Korea) prepared via chemical vapor deposition. The epoxy was diglycidyl ether of bisphenol A (YD-115, Kukdo Chemical, Korea), and the curing agent was polyamidoamine (G-A0533, Kukdo Chemical, Korea). The reagents used for the acid treatment were nitric acid (60-62\%, Junsei Chemical, Japan), sulfuric acid (95\%, Junsei Chemical, Japan), acetone (99.5\%, Dae Jung Chemical, Korea), and ethanol (99.5\%, Aldrich, USA). The acid treatment of the CNTs and the fabrication of the CNT/epoxy/ basalt composites were performed using the procedure described in the previous article. For the double cantilever beam (DCB) fracture specimens (width $25 \mathrm{~mm}$ and length $200 \mathrm{~mm}$ ), an initial delamination was created through the insertion of a $50 \mathrm{~mm}$ Kap ton film (thickness: $13 \mu \mathrm{m}$ ) between the fourth and fifth plies. The fracture specimens were constantly immersed in sterile filtered seawater (S-9148, Sigma-Aldrich, USA) for up to six months.

The fracture tests were performed using the DCB fracture specimens (width $25 \mathrm{~mm}$ and length $200 \mathrm{~mm}$ ) at a loading rate of $0.5 \mathrm{~mm} / \mathrm{min}$ in accordance with the guidelines set in ASTM D 5528-01 [11]. After the initial delamination increased to a predetermined extent, the applied displacement was reduced and then increased again to induce further delamination. This process was repeated more than ten times until the delamination increased to more than $30 \mathrm{~mm}$. A minimum of five flexural and fracture tests were performed to ensure the reliability of the test results.

\section{Results and Discussion}

The mode I fracture toughness $\left(G_{I c}\right)$ was determined using the compliance calibration method, as follows:

$$
G_{I C}=\frac{n P_{c r} \delta}{2 b a},
$$

where $a$ represents the crack length, $b$ is the specimen width, $\delta$ is the load-point displacement, and $P_{c r}$ is the fracture load. The $n$ value in Eq. (1) represents a slope in the plot of the corrected compliance versus crack length in a log-log scale. The compliance changes according to the crack propagation for the dry and seawater-absorbed CNT/epoxy/basalt composites were determined using $P-\delta$ curves, where the compliance for each crack length was determined by measuring the inverse slope of the corresponding unloading line. Fig. 1 presents the variations in the compliance with crack increases in a $\log$ - $\log$ scale for both composites. In the figure, it can be seen that the difference in compliance between both composites is not significant. However, the compliance of the seawater-absorbed CNT/epoxy/basalt composites is larger than that of the dry CNT/epoxy/basalt composites at the same crack length. It can also be seen in Fig. 1 that there is a linear relationship between $\log C$ and $\log a$ for each specimen. In particular, the $n$ values that were determined as the slopes of the plots for each specimen differed to each other. Specifically, the $n$ values of the dry and seawater-absorbed $\mathrm{CNT} /$ epoxy/basalt composites were 2.48 and 2.15 , respectively.

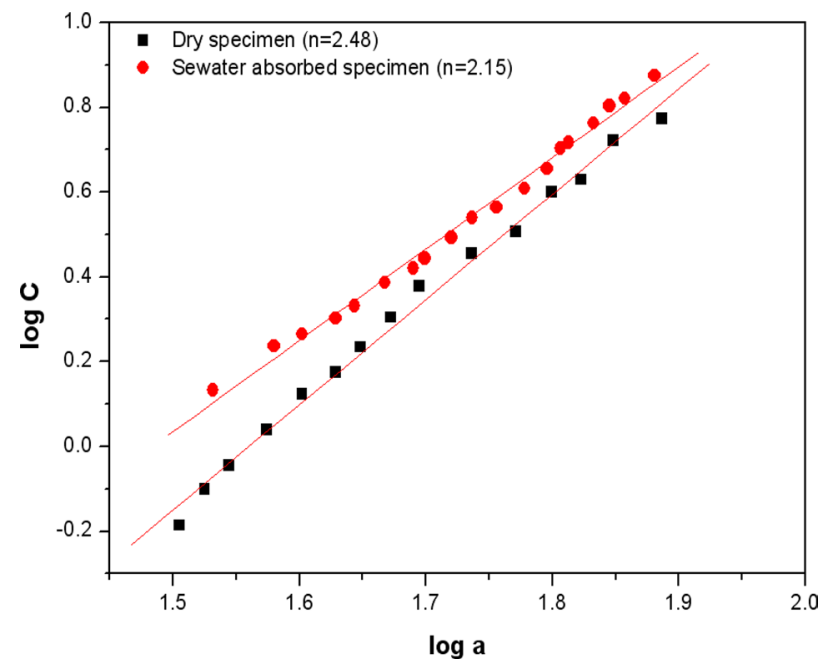

Fig. 1. Comparison of the $n$ values in a log-log scale.

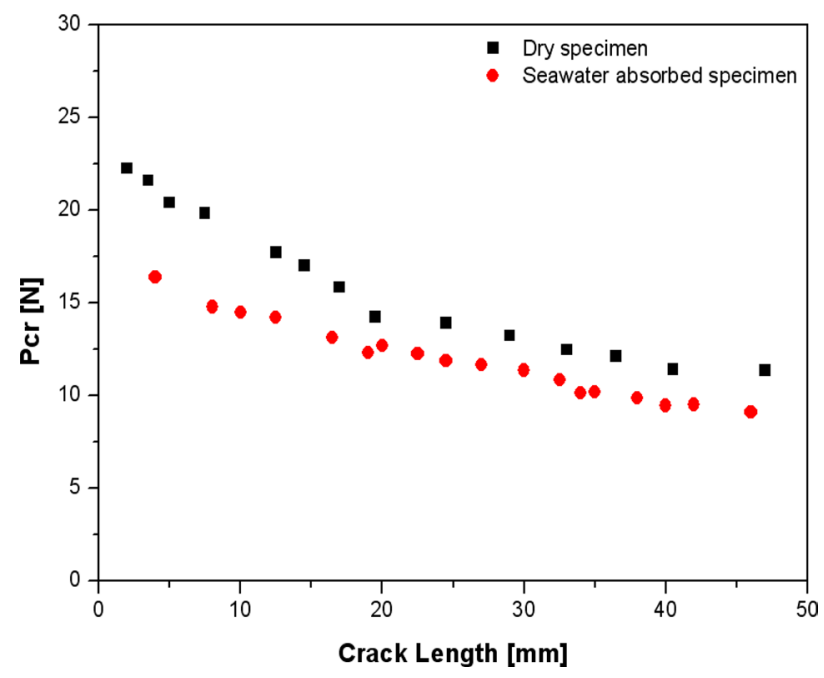

Fig. 2. Comparison of $P_{c r}$ as a function of crack length.

Fig. 2 shows the $P_{c r}$ variations during the crack propagation for the dry and seawater-absorbed CNT/epoxy/basalt composites. As shown in the figure, the $P_{c r}$ decreased as the crack length increased for both composites. Interestingly, contrary to the compliance case, the $P_{c r}$ of the seawater-absorbed CNT/epoxy/ basalt composites was smaller than that of the dry CNT/epoxy/ basalt composites at the same crack length, which indicates that the seawater absorption decreases the adhesion strength between the layers of the CNT/epoxy/basalt composites.

The mode I fracture toughness $\left(G_{I c}\right)$ of the seawater-absorbed CNT/epoxy/basalt composites determined using Eq. (1) was compared with that of the dry CNT/epoxy/basalt composites. Fig. 3 shows a comparison of the variations in the $G_{I c}$ according to the crack length for both composites. It can be seen in the figure that the $G_{I c}$ varies over a limited range according to the crack length for both composites. It can also be seen that the $G_{I c}$ of the seawater-absorbed CNT/epoxy/basalt composites was approximately $20 \%$ smaller than that of the dry CNT/epoxy/basalt composites at the same crack length. Specifically, the averaged 


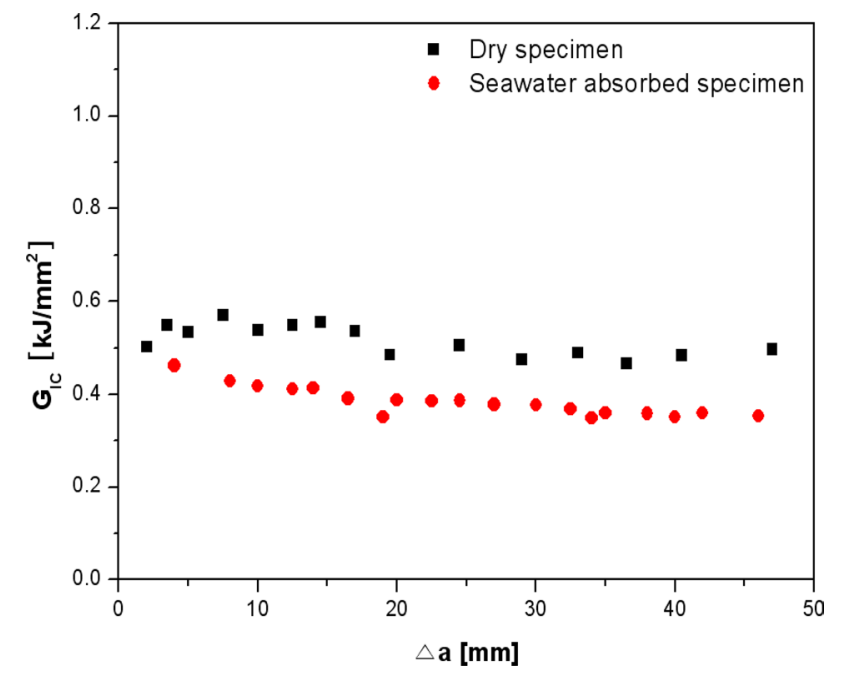

Fig. 3. Comparison of $G_{k}$ values as a function of the crack length.

values of $G_{I c}$ for the dry and seawater-absorbed CNT/epoxy/ basalt composites were $0.52 \mathrm{~kJ} / \mathrm{mm}^{2}$ and $0.4 \mathrm{~kJ} / \mathrm{mm}^{2}$, respectively. It is believed that the decreased fracture toughness of the seawater-absorbed CNT/epoxy/basalt composites is attributable to the poor interfacial interactions between the CNTs and the epoxy matrix, which resulted from the swelling of the epoxy matrix during the seawater absorption.

\section{Conclusions}

This study investigated the effect of seawater absorption on the fracture toughness of CNT/epoxy/basalt composites. Overall, the fracture properties of the CNT/epoxy/basalt composites were significantly affected by seawater absorption. The compliance of the seawater-absorbed CNT/epoxy/basalt composites was larger than that of the dry CNT/epoxy/basalt composites at the same crack length, while the fracture load exhibited the opposite behavior. The fracture toughness of the seawater-absorbed CNT/epoxy/basalt composites was approximately $20 \%$ less than that of the dry CNT/epoxy/basalt composites. The decrease in the fracture toughness of the seawater-absorbed CNT/epoxy/basalt composites was attributed to the weakening of the interfacial bonding between the CNTs and epoxy due to the swelling of the epoxy matrix.

\section{Acknowledgements}

This work was financially supported by the national R\&D project titled "Development of Energy Utilization of Deep
Ocean Water", which was supported by the Korean Ministry of Land, Traffic and Maritime Affairs.

\section{References}

[1] Singha K. A short review on basalt fiber, Int J Text Sci, 1, 19 (2012). http://dx.doi.org/10.5923/j.textile.20120104.02.

[2] Zhang Y, Yu C, Chu PK, Lv F, Zhang C, Ji J, Zhang R, Wang $\mathrm{H}$. Mechanical and thermal properties of basalt fiber reinforced poly(butylene succinate) composites. Mater Chem Phys, 133, 845 (2012). http://dx.doi.org/10.1016/j.matchemphys.2012.01.105.

[3] Xin SB, Liang XP, Liu HW, Zhong ZL. Wear properties of basalt fibers reinforced composites. Key Eng Mater, 368-372, 1010 (2008). http://dx.doi.org/10.4028/www.scientific.net/KEM.368-372.1010.

[4] Varley RJ, Tian W, Leong KH, Leong AY, Fredo F, Quaresimin $M$. The effect of surface treatments on the mechanical properties of basalt-reinforced epoxy composites. Polym Compos, 34, 320 (2013). http://dx.doi.org/10.1002/pc.22412.

[5] Chairman CA, Babu SPK, Natarajan S. Corrosion and abrasive wear studies of basalt fabric reinforced epoxy composites. 27th International Conference on Surface Modification Technologies, Chennai India (2013).

[6] Kim MT, Rhee KY. Flexural behavior of carbon nanotube-modified epoxy/basalt composites. Carbon Lett, 12, 177 (2011). http:// dx.doi.org/10.5714/CL.2011.12.3.177.

[7] Tehrani M, Boroujeni AY, Hartman TB, Haugh TP, Case SW, AlHaik MS. Mechanical characterization and impact damage assessment of a woven carbon fiber reinforced carbon nanotube-epoxy composite. Compos Sci Technol, 75, 42 (2013). http://dx.doi. org/10.1016/j.compscitech.2012.12.005.

[8] Sanchez M, Campo M, Jimenez-Suarez A, Urena A. Effect of the carbon nanotube functionalization on flexural properties of multiscale carbon fiber/epoxy composites manufactured by VARIM. Composites B, 45, 1613 (2013). http://dx.doi.org/10.1016/j.compositesb.2012.09.063.

[9] Ashrafi B, Guan J, Mirjalili V, Zhang Y, Chun L, Hubert P, Simard B, Kingston CT, Bourne O, Johnston A. Enhancement of mechanical performance of epoxy/carbon fiber laminate composites using single-walled carbon nanotubes. Compos Sci Technol, 71, 1569 (2011). http://dx.doi.org/10.1016/j.compscitech.2011.06.015.

[10] Kim MT, Rhee KY, Park SJ, Hui D. Effects of silane-modified carbon nanotubes on flexural and fracture behaviors of carbon nanotube-modified epoxy/basalt composites. Composites B, 43, 2298 (2012). http://dx.doi.org/10.1016/j.compositesb.2011.12.007.

[11] ASTM D 5528-01: Standard test method for mode I interlaminar fracture toughness of unidirectional fiber-reinforced polymer matrix composites, ASTM International, West Conshohocken, PA (2007). 